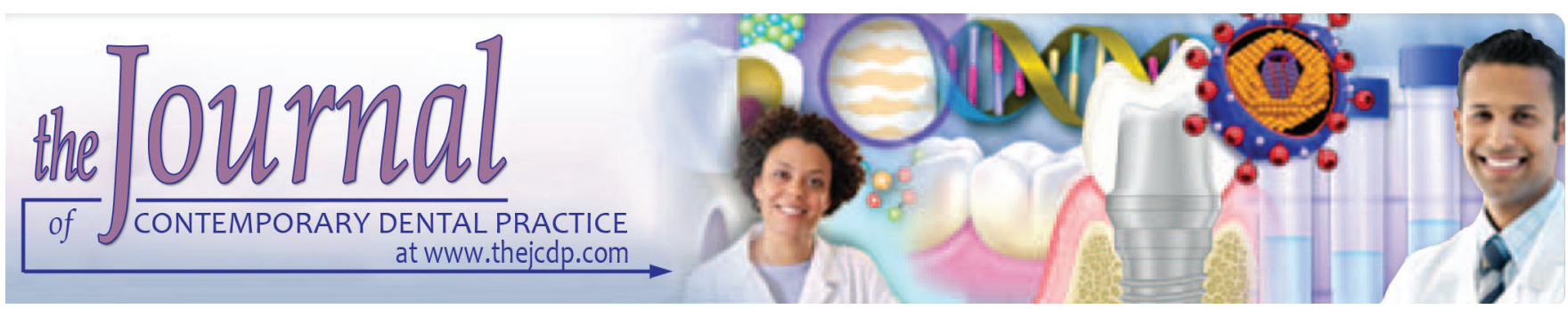

\title{
Institutional Ethics Committee Regulations and Current Updates in India
}

\author{
${ }^{1}$ Amit V Mahuli, ${ }^{2}$ Simpy A Mahuli, ${ }^{3}$ Shankargouda Patil, ${ }^{4}$ Shilpa Bhandi
}

\begin{abstract}
Aim: The aim of the review is to provide current updates on regulations for ethics committees and researchers in India.
\end{abstract}

Background: Ethical dilemmas in research since time immemorial have been a major concern for researchers worldwide. The question "what makes clinical research ethical" is significant and difficult to answer as multiple factors are involved.

Review results: The research involving human participants in clinical trials should follow the required rules, regulations, and guidelines in one's own country. It is a dynamic process, and updates have to be learned by researcher and committee members. The review highlights the ethical regulation from the Drug Controller General of India, Clinical Trial Registry of India, and Indian Council of Medical Research guidelines.

Conclusion: In this article, the updates on Indian scenario of the Ethical Committee and guidelines are compiled.

Clinical significance: The review comes handy for clinical researchers and ethics committee members in academic institutions to check on the current updates and keep abreast with the knowledge on regulations of ethics in India.

Keywords: Audiovisual consent, Biomedical research, Ethics and informed consent.

How to cite this article: Mahuli AV, Mahuli SA, Patil S, Bhandi S. Institutional Ethics Committee Regulations and Current Updates in India. J Contemp Dent Pract 2017;18(8):738-741.

Source of support: Nil

Conflict of interest: None

\footnotetext{
1,2Department of Public Health Dentistry, NIMS Dental College Jaipur, Rajasthan, India

${ }^{3}$ Division of Oral Pathology, Department of Maxillofacial Surgery and Diagnostic Sciences, College of Dentistry, Jazan University Jazan, Kingdom of Saudi Arabia

${ }^{4}$ Department of Restorative Dental Sciences, College of Dentistry, Jazan University, Kingdom of Saudi Arabia

Corresponding Author: Amit V Mahuli, Department of Public Health Dentistry, NIMS Dental College, Jaipur, Rajasthan, India Phone: +918107960145, e-mail: amitmahuli@gmail.com
}

\section{INTRODUCTION}

Ethical dilemmas in research since time immemorial have been a major concern for researchers worldwide. The question "what makes clinical research ethical" is significant and difficult to answer as multiple factors are involved. With the enormous amount of literature and guidelines available online, it becomes very tricky to follow the right ones. The basic ethical principles are autonomy, justice, beneficence, and nonmalfeasance for research. The current ethical principles assess the risk-benefit ratio of the clinical trial so that the results can be generalized and extrapolated to the population as a whole. ${ }^{1}$

The regulations unique to the country of research are mandatory to follow. Table 1 lists few important ethical guidelines that a researcher and ethics committee member should be aware of..$^{2-7}$ The Indian Council of Medical Research ${ }^{7}$ has proposed ethical guidelines for Biomedical Research in India. The majority of the guidelines explicitly mention about the clinical trial using the newly found drug for human participants and any intervention, such as drugs, surgical procedures, preventive measures, lifestyle modifications, devices, educational or behavioral treatment, and rehabilitation strategies. In academic institutions, the protocol has to meet various screening stages as shown in Flow Chart 1 before assessment of ethics committee.

\section{REGULATIONS IN INDIAN PERSPECTIVE}

In India, it is mandatory to take ethical clearance for a clinical trial on human participants from a registered ethical committee under Rule 122DD and informed consent according to Appendix VIII of Schedule Y of Drugs and Cosmetics Act, 1940 and Rules 1945 regulated by the Central Drug Standard Control Organization (CDSCO), Office of Drug Controller General India (DCGI), Directorate General of Health Services, and Ministry of 
Institutional Ethics Committee Regulations and Current Updates in India

Table 1: List of applicable ethical guidelines for research in human participants

\begin{tabular}{llc}
\hline Guidelines & Source & Year with update \\
\hline Nuremberg code & $\begin{array}{l}\text { Nuremberg military tribunal decision in the United } \\
\text { States v Brandt }\end{array}$ & 1947 \\
Belmont report & $\begin{array}{l}\text { National commission for the protection of human } \\
\text { subjects of biomedical and behavioral research } \\
\text { The WHO technical report series }\end{array}$ & 1979 \\
$\begin{array}{l}\text { Guidelines for good clinical practice } \\
\begin{array}{l}\text { Standards and operational guidance for ethics review } \\
\text { of health-related research with human participants }\end{array}\end{array}$ & World Health Organization \\
$\begin{array}{l}\text { Ethical principles of medical research involving human } \\
\text { participants }\end{array}$ & World Medical Association: Declaration of Helsinki & 2011 \\
$\begin{array}{l}\text { Ethical guidelines for biomedical research on human } \\
\text { participants }\end{array}$ & Indian Council of Medical Research 2013 \\
\hline
\end{tabular}

Flow Chart 1: Functioning of institutional review boards (IRBs)/ethics committees in academic setup

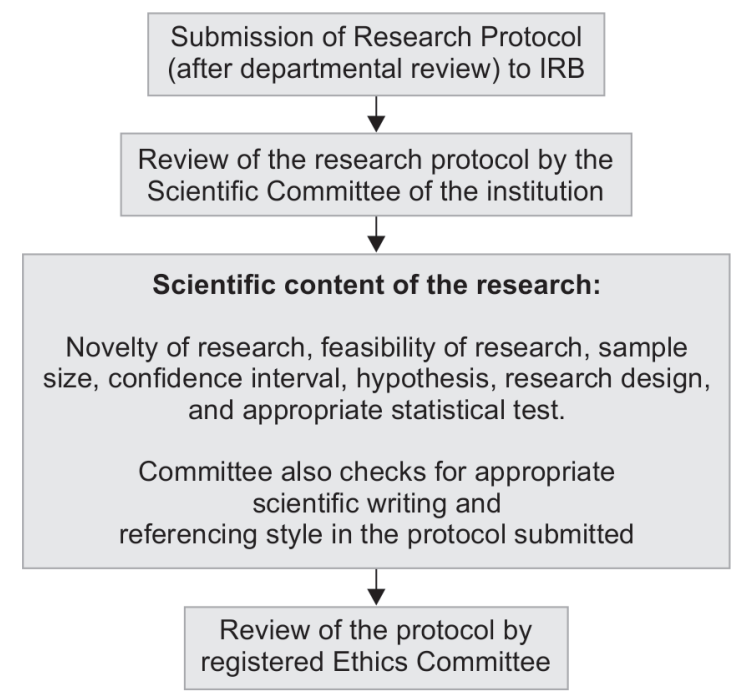

Health and Family Welfare, Central Government, India. As per Schedule Y, a freely given written, informed consent of individuals participating in the research trial is mandatory. Audiovisual (AV) recording of the informed consent process has been made mandatory for clinical trials, following the Supreme Court decision dated October 21, 2013, as per the DCGI office order dated November 19, 2013. ${ }^{8,9}$

Trial registration in the Clinical Trial Registry - India (CTRI) is mandatory by the DCGI (www.cdsco.nic. in). The CTRI was first launched on July 20, 2007; since then, more than 3,300 trials have been registered until January 2013. The CTRI, in turn, is a part of the World Health Organization (WHO)'s International Clinical Trials Registry Platform, so all the trials add to the global pool of data. ${ }^{10}$

\section{FUNCTIONS AND FORMATION OF THE ETHICS COMMITTEE IN INDIA}

- The institutional ethics committee has to prepare a constitution, standard operating procedures, conditions for member appointment, the offices, and the quorum requirements. ${ }^{11}$
- Ethics committee shall review the research protocol as per Schedule Y and Good Clinical Practice Guidelines for Clinical Trials in India to safeguard the right, wellbeing, and safety of the trial participants.

- In the case of any adverse event during the clinical trial, the ethics committee shall analyze and forward its opinion as per schedule $\mathrm{Y}$.

- Ethics committee shall cooperate with the inspectors and officials authorized by the Central Drugs Standard Control Organization to enter its premises to inspect any record, data, or any document related to clinical trial and provide adequate replies to any query.

- The committee shall maintain all the information regarding the clinical trial for at least 5 years after the completion of the trial.

- The ethics committee registration is valid for 3 years. The committee should inform in writing to the licensing authority in case of any changes in the ethics committee. Licensing authority has the right to issue show cause notice in the event of any complaints and wrongdoing. The authority has the right to cancel the registration of the committee.

- Ethics committee should have a minimum of 7 members and a maximum of 15 members, which includes a chairperson (independent of an institution or outside the institution), one member secretary, clinicians (medical and scientific), legal expert, social scientist, and layperson.

- For clearing the protocol for a clinical trial, the ethics committee should have the quorum of at least five members: Basic scientist, legal expert, clinician, social scientist, and layperson.

- The principal investigator and the Ethical Committee members are expected to have adequate knowledge on Good Clinical Practice guidelines for clinical trials in India. Members representing ethics committee should have a postgraduate degree, possess sufficient scientific knowledge, and should be aware of their roles and responsibilities as board members. 
- There should be no conflict of interest while decision making on the research protocol from any of the committee members. All members should declare their conflict of interest.

- The committee can invite specialist and experts in the case of discussion related to the topic of their expertise, but the invited experts will not have the right of voting.

\section{RESPONSIBILITIES OF ETHICS COMMITTEE}

- The novelty of research and social value: The study should add to the existing knowledge or should help in innovation for the new knowledge and should benefit the society and humanity by enlarge.

- Scientifically sound: The research should be scientifically valid sound study design, appropriate statistical analysis, the feasibility of the study, and precise scientific objective.

- Fair selection of the study samples: Selection of the vulnerable group for research with risky outcomes is a major ethical problem. Appropriate selection of the sample based on the merit of the study and justice to the sample is of most importance.

- Favorable risk-benefit ratio: Maximum benefit and minimum risk care should be taken to have minimal risk to the patient in comparison to the benefit. The benefit is assessed on the total good from the research to the individual and the society. Patients should not be exploited.

- Independent review of the research protocol: Review of the protocol for possible conflict of interest, the source of funding, and other financial details are to be thoroughly assessed. There should also be review of research design of the trial and risk to the population recruited in the trial.

- Informed consent: It should be taken from every sample recruited in the study; it should be voluntary, legal, and comprehending and should be recorded and maintained as per regulations of the country.

- Respect for potential and enrolled participants in the study: Autonomy of the participants involved in research is very crucial; the participants need to be informed of the risk and benefits involved in the trial, and they have the right to withdraw from the study at any given time.

\section{AUDIOVISUAL INFORMED CONSENT}

The clinical trials on new drugs have strict regulations in India; Drug \& Cosmetic Rule 1945 being amended from time to time under Schedule Y of the rules. The clinical trials are to be conducted with approved protocols, and the Good Clinical Practice Guidelines published by CDSCO and Directorate General of Health Services, Government of India. ${ }^{9}$

Honorable Supreme Court of India, CDSCO vide F. No. GCT/20/SC/Clin./2013 DCG1 dated November 19, 2013, has categorically stated that all clinical trials will have to follow AV consent in addition to the written informed consent. The statement should clearly explain the study participants about the purpose of research, duration, the procedure/intervention involved, the possible risk involved, benefit to the participant, referrals in case of emergency, and compensation to the study participants. The investigator should maintain confidentiality regarding the participants involved in the trial. The consent has to be explained in the language the patient understands the best, in simple and not technical words. The consent has to be understood, voluntary, and the participant has the right to withdraw from the study anytime.

\section{METHOD OF RECORDING}

The investigator will identify the participant involved, the language they understand, and protocol of the study. A study team member can help as interpreter if the language is not known. The photo ID of the participant is documented to identify the participant. The video camera for the AV recording should be of adequate capability to simultaneously capture the facial details of the participant, impartial witness (if any), and investigator / authorized person present during the consent process. The AV recording should be conducted in a room conducive to the recording of disturbance-free audio and video of the consent process. During the videography process, care should also be taken not to include unrelated persons/patients at the hospital within the field of vision. Poor video or audio quality may not sever the purpose of consent; care should be taken to use the camera with good resolution and mic with good quality. The AV recordings of consent have to be preserved for a minimum period of 5 years after the completion of the study.

\section{LATEST UPDATES}

- No permission for conduct of clinical trial intended for academic purposes with respect to approved drug formulation shall be required for any new indication or new route of administration or new dose or new dosage form where the trial is approved by the ethics committee, the data generated are not intended for submission to the licensing authority. The ethics committee, however, has to inform the licensing authority about the study updates. ${ }^{12,13}$ 
- The participants have to be notified about the intervention failure regarding therapeutic effect in the clinical trial involving new drugs.

- The participants should be disclosed about placebocontrolled trials that placebo administered will not have any therapeutic effect.

\section{CONCLUSION}

In the current scenario, researchers and ethics committees have to keep track of the changes in the rules, regulations, and amendments as this is a dynamic process. There can be more seminars and workshops to discuss the updates and latest guidelines. With the number of clinical trials increasing, there will be more complex ethical issues to handle and to learn from different situations. The CDSCO is providing with all updates on their website.

\section{CLINICAL SIGNIFICANCE}

The review comes handy for clinical researchers and ethics committee members in academic institutions to check on the current updates and keep abreast with the knowledge on regulations of ethics in India.

\section{REFERENCES}

1. Emanuel EJ, Wendler D, Grady C. What makes clinical research ethical? JAMA 2000 May;283(20):2701-2711.

2. Nuremberg Military Tribunal. The Nuremberg Code. J Am Med Assoc 1996 Nov;276(20):1691.

3. National Commission for the Protection of Human Subjects of Biomedical and Behavioral Research. The Belmont Report. Washington (DC): US Government Printing Office; 1979.
4. World Health Organization. Guidelines for good clinical practice (GCP) for trials on pharmaceutical products. Vol. 850. Geneva: WHO Technical Report Series; 1995. p. 97-137.

5. World Health Organization. Standards and operational guidance for ethics review of health-related research with human participants. Geneva: World Health Organization; 2011.

6. World Medical Association. World Medical Association Declaration of Helsinki: ethical principles for medical research involving human subjects. Seoul: World Medical Association; 2008. [cited 2016 Aug 23]. Available from: http:/ / www.wma. net/en/30publications/10policies/b3.

7. ICMR. Ethical guidelines for biomedical research on human participants. New Delhi: ICMR; 2006. [cited 2016 Aug 26]. Available from: http:/ / www.icmr.nic.in/ethical_guidelines.pdf.

8. CDSCO. Drugs and Cosmetics Act, 1940. As Amended up to the 30th June. India: CDSCO; 2005. [cited 2016 Aug 26]. Available from: http://www.cdsco.nic.in/forms/contentpage1.aspx?lid=1888.

9. CDSCO. Draft Guidelines on Audio-Visual Recording of Informed Consent Process in Clinical Trial. India: CDSCO; 2014. [cited 2016 Aug 26]. Available from: http:/ / www.cdsco. nic.in/writereaddata/Guidance_for_AV\%20Recording_09. January.14.pdf.

10. Clinical Trial Registry India. [cited 2016 Aug 26]. Available from: http:/ / www.ctri.nic.in/Clinical-trials/login.php.

11. CDSCO. Indian GCP Guidelines. New Delhi: CDSCO; 2004. [cited 2016 Aug 24]. Available from: http:/ /www.cdsco.nic. in/html/GCP1.html.

12. Gazette Notification published on 6th January, 2016. G.S.R. 611(E). 2016. [cited 2016 Aug 24]. Available from: http:/ / www. cdsco.nic.in/writereaddata/GSR\%2011(E)\%20dated $\% 20$ 06_01_2016.pdf.

13. Gazette Notification published on 31st July, 2015, G.S.R. 11(E). 2015. [cited 2016 Aug 24]. Available from: http:/ / www.cdsco. nic.in/writereaddata/Gazette $\% 20$ Notification $\% 2031 \% 20$ July\%202015.pdf. 